\title{
Appropriate Source Control and Antifungal Therapy are Associated with Improved Survival in Critically Ill Surgical Patients with Intra-abdominal Candidiasis
}

\author{
Ting $\mathrm{Yan}^{1} \cdot$ Shuang-Ling $\mathrm{Li}^{1} \cdot \mathrm{Hai}-\mathrm{Li} \mathrm{Ou}^{2} \cdot$ Sai-Nan $\mathrm{Zhu}^{3} \cdot$ \\ Lei Huang ${ }^{2}$ - Dong-Xin Wang ${ }^{1}$ (I)
}

Published online: 21 January 2020

(C) The Author(s) 2020

\begin{abstract}
Background Intra-abdominal candidiasis (IAC) is the predominant type of invasive candidiasis with high mortality in surgical intensive care patients. The purpose of this study was to investigate the impact of appropriate source control and antifungal therapy on the outcomes of critically ill surgical patients with IAC.

Methods This was a retrospective single-center cohort study. Adult surgical patients who were admitted to the intensive care unit and diagnosed with IAC from January 1, 2003, to December 31, 2016, were enrolled. The patients' data including risk factors of IAC, infection-related information, antifungal treatment and 30-day outcomes were collected. The primary endpoint was 30-day mortality. A COX proportional hazards model was used to analyze the association between appropriate treatment and 30-day survival.

Results A total of 82 patients were included in the analysis. Of these, 45 (54.9\%) were complicated with septic shock at IAC diagnosis. Types of IAC included peritonitis (61.0\%), intra-abdominal abscesses $(23.2 \%)$ and biliary tract infections (15.9\%). Of the included patients, $53(64.6 \%)$ received appropriate source control and $44(53.7 \%)$ appropriate antifungal therapy. Compared with patients with neither of these treatments, appropriate source control (HR $0.08,95 \%$ CI $0.02-0.30 ; P<0.001$ ), appropriate antifungal therapy (HR $0.14,95 \%$ CI $0.04-0.55 ; P=0.005$ ), and a combination of these treatments (HR $0.02,95 \%$ CI $0.00-0.08 ; P<0.001$ ) were associated with reduced risk of death within 30 days after IAC diagnosis.

Conclusion For critically ill surgical patients with IAC, both appropriate source control and appropriate antifungal therapy were associated with reduced risk of 30-day mortality, and the protective effects of the two appropriate treatments were additive.
\end{abstract}

Dong-Xin Wang

wangdongxin@hotmail.com

1 Department of Anesthesiology and Critical Care Medicine, Peking University First Hospital, No.8 Xishiku Street, Beijing 100034, China

2 Department of Clinical Laboratory, Peking University First Hospital, Beijing, China

3 Department of Biostatistics, Peking University First Hospital, Beijing, China

\begin{tabular}{ll}
\multicolumn{2}{l}{ Abbreviations } \\
IAC & Intra-abdominal candidiasis \\
ICU & Intensive care unit \\
IC & Invasive candidiasis \\
MIC & Minimum inhibitory concentration \\
APACHE II & Acute physiology and chronic health \\
& evaluation II \\
SOFA & Sepsis-related organ failure assessment \\
COPD & Chronic obstructive pulmonary disease \\
BMI & Body mass index \\
MDRO & Multidrug-resistant organism
\end{tabular}




\section{Introduction}

Intra-abdominal candidiasis (IAC) is the most common type of deep-seated candidiasis in critically ill surgical patients, which accounts for 34-59\% of invasive candidiasis (IC) $[1,2]$ and $10-20 \%$ of intra-abdominal infections [3]. IAC is a life-threatening complication with high morbidity and mortality. According to the epidemiological data over the last decades, the mortality rate of patients with IAC was about $25-40 \%$; for those combined with septic shock, the mortality rate was up to 60\% [4-6]. Unfortunately, IAC remains poorly understood when compared with candidemia [7-9]. Previous studies showed that, for patients with candidemia, early antifungal therapy and timely source control improve survival [10-13]. However, results regarding the treatment for IAC are insufficient and conflicting. Some authors reported that delayed or insensitive initial antifungal therapy was an independent risk factor of 30-day mortality in patients with IAC [6, 14], whereas some others did not find an association between the antifungal therapy and 30-day mortality [15]. On the other hand, available evidence supports the use of early source control in managing IAC [6, 14-16].

In the present study, we focused on IAC in critically ill surgical patients in whom surgical interventions to reduce microbiological burden are strongly indicated and the effect of antifungal therapy is still expected to be determined. The purpose of this retrospective cohort study was to explore the effects of appropriate treatments (including source control and antifungal therapy) on 30-day survival in critically ill surgical patients with IAC.

\section{Materials and methods}

This was a single-center retrospective cohort study. The study protocol was approved by the Clinical Research Ethics Committee of Peking University First Hospital (2017-1303). Because of the retrospective and observational nature of the study, the local Ethics Committee agreed to exempt written informed consent.

\section{Patients}

This study screened all the patients who were admitted to the surgical ICU of Peking University First Hospital between January 1, 2003, and December 31, 2016. The inclusion criteria were as follows: (1) age over 18 years; (2) confirmed intra-abdominal infection after abdominal surgery or required surgical intervention; (3) IAC diagnosed during ICU stay. Patients who met any of the following criteria were excluded: (1) neutropenia (absolute neutrophil count $<500$ cells $/ \mu \mathrm{L}$ ); (2) recipient of bone marrow or solid organ transplantation; (3) chemotherapy in the last 6 months; (4) receiving immunosuppressants or systemic steroids (prednisone equivalent $\geq 20 \mathrm{mg} /$ day) within 7 days prior to ICU admission; (5) acute pancreatitis; (6) primary peritonitis; (7) documented invasive candidiasis within 6 months, or received systemic antifungal therapy within 14 days; or (8) incomplete data.

\section{Diagnosis of intra-abdominal candidiasis}

According to the 2013 European consensus [6, 9, 17], an episode of IAC was diagnosed in one of the following four conditions: (1) Candida detection by direct microscopy examination or growth in culture from purulent or necrotic intra-abdominal specimens obtained during surgery or by percutaneous aspiration; (2) Candida growth from bile, intra-biliary ducts devices, and biopsy of intra-abdominal organs; (3) Candida growth from blood cultures in clinical setting of secondary and tertiary peritonitis in the absence of any other pathogen; (4) Candida growth from drainage tubes only if placed less than $24 \mathrm{~h}$ before the cultures.

To ensure the accuracy of IAC diagnosis, two researchers determined IAC independently. In case of a difference between the two researchers, final agreement was achieved by rechecking the records and full discussion with a senior physician. Documented IAC were identified by reviewing hospital medical records. The clinical specimens suspected of yeast infection were primarily cultured in the Sabouraud agar plates, and suspected colonies were screened for further identification. The identification of different yeasts to species level was performed by the CHROMagar ${ }^{\mathrm{TM}}$ Candida (BD Difco, Detroit, MI) and the Vitek 2 Compact automated system (BioMérieux, Marcy l'Etoile, France) with YST card. The equivocal results were confirmed by sequencing of the internal transcribed spacer (ITS) region. Antifungal susceptibility testing was performed with the $\mathrm{ATB}^{\mathrm{TM}}$ FUNGUS 3 stripe (BioMérieux, Marcy l'Etoile, France), in which the susceptibility testing for echinocandin was not included, so the susceptibility results for echinocandin were unavailable. The results interpretation was made following the NCCLS document M27-A [18].

\section{Appropriate treatment}

Appropriate source control was defined as adequate source control within 5 days since the positive cultures were obtained. Adequate abdominal source control was defined as: (1) adequate drainage of infected fluid collections, (2) debridement of infected necrotic tissue, and (3) definitive intervention to correct anatomic derangements resulting in ongoing microbial contamination and restore optimal 
function [19]. The surgical interventions could be combined with irrigation, but simple irrigation couldn't be judged as adequate source control.

Appropriate initial antifungal therapy was considered if the following conditions were satisfied: (1) early: antifungal treatment started within 5 days since the positive cultures were obtained [14]; (2) active: the infecting organism was ultimately shown to be susceptible, and the dose of antifungal agent was adequate [20]. The echinocandins were assessed as susceptible for all Candida species. The following antifungal doses were considered adequate: (1) for fluconazole-susceptible Candida isolates (MIC $\leq 8$ $\mathrm{mg} / \mathrm{L}$ ), a minimal daily dose of $400 \mathrm{mg}$ was considered appropriate. For fluconazole-susceptible dose-dependent isolates (SDD; MIC 16-32 mg/L), a minimal daily dose of $800 \mathrm{mg}$ was considered adequate. For patients with a calculated creatinine clearance $<50 \mathrm{~mL} / \mathrm{min}$, a daily dose of fluconazole of $50 \%$ of the normal dose was considered adequate based on standard dosing adjustments made in renal dysfunction; (2) $\geq 0.5 \mathrm{mg} / \mathrm{kg}$ of amphotericin B deoxycholate once daily; $\geq 3 \mathrm{mg} / \mathrm{kg}$ of amphotericin B lipid formulations once daily; (3) caspofungin $70 \mathrm{mg}$ loading dose followed by $50 \mathrm{mg} /$ day (or $35 \mathrm{mg}$ once daily for patients with significant liver impairment), micafungin $\geq 100 \mathrm{mg} /$ day (formulary echinocandin beginning in 2008); (4) $6 \mathrm{mg} / \mathrm{kg}$ of voriconazole twice daily followed by $\geq 3 \mathrm{mg} / \mathrm{kg}$ twice daily [20].

\section{Data collection}

Patients' data were screened through the electronic medical record system of the hospital, and eligible patients were identified according to the inclusion/exclusion criteria.

For included patients, detailed information was collected. The baseline data included demographic parameters, surgical diagnosis, comorbidities, classical risk factors for IAC, as well as the assessment of disease severity. The acute physiology and chronic health evaluation (APACHE) II score and the sepsis-related organ failure assessment (SOFA) score were calculated for each patient within $24 \mathrm{~h}$ prior to the collection of cultures indicating IAC. Organ failure was diagnosed when the SOFA score of this organ was greater than 2 [21]. Septic shock was diagnosed according to the third international consensus definitions [22]. The characteristics of IAC included surgical conditions, type of IAC, Candida species, concomitant candidemia, and bacterial co-infection. IAC-related treatments included source control (surgical intervention, percutaneous drainage, and the appropriateness of source control) and initial antifungal therapy (type of antifungal medication and the appropriateness of antifungal therapy).

The primary outcome was 30-day survival after the diagnosis of IAC, including all-cause 30-day mortality and the time to death or loss to follow-up within 30 days. The secondary outcome was mortality during hospitalization.

\section{Statistical analysis}

Patients were divided into four treatment groups according to the combination of appropriate source control and antifungal therapy. Data were tested for normality using the Kolmogorov-Smirnov test. Continuous variables with normal distribution were compared with one-way ANVOA and post hoc student $t$ test; continuous variables with nonnormal distribution or ranked data were compared with Kruskai-Wallis $H$ test and post hoc Mann-Whitney $U$ analysis. Categorical variables were compared with Fisher's exact test and post hoc Chi-squared test or Fisher exact test. Survival data were analyzed with the KaplanMeier estimator, with differences between groups assessed by the log-rank test. Factors in association with 30-day survival were identified using a Cox proportional hazards model; variables with a $P$ value of $<0.10$ in univariate analyses were included in a multivariate model (backward). Two-sided $P$ values of $<0.05$ were regarded as statistically significant. For multiple comparisons among the four groups, $P$ values of $<0.05 / 6=0.0083$ were considered statistically significant (Bonferroni correction). Statistical analyses were performed with SPSS statistical package version 25.0 (IBM SPSS Inc, Chicago, IL, USA).

\section{Results}

\section{Patients}

From January 1, 2003, to December 31, 2016, 12,127 patients were admitted to the surgical ICU. Of these, 94 (7.8\%o) were diagnosed with IAC, 82 met the inclusion/ exclusion criteria and were included in final analysis (Fig. 1). Of the included patients, the median age was 70.1 years, $44(53.7 \%)$ were male, the mean APACHE II score was $17.7 \pm 6.9,45(54.9 \%)$ presented with septic shock at the time of the diagnosis, and $59(72.0 \%)$ had at least one organ failure during hospital stay. The baseline characteristics and the risk factors for IAC are summarized in Table 1.

In our patients with IAC, $50(61.0 \%)$ had secondary or tertiary peritonitis, 19 had $(23.2 \%)$ abdominal abscesses, and $13(15.9 \%)$ had hepatobiliary system infections. A total of 84 Candida strains were isolated from 82 patients. Two (2.4\%) patients suffered from polyfungal IAC. Of the isolated Candida strains, 83 had antifungal susceptibility testing results; $3(3.6 \%)$ of $C$. glabrata and 1 (1.2\%) of $C$. albicans were resistant to fluconazole; the others were susceptible to azoles. Bacterial co-infections and 
Fig. 1 Flow diagram of the study

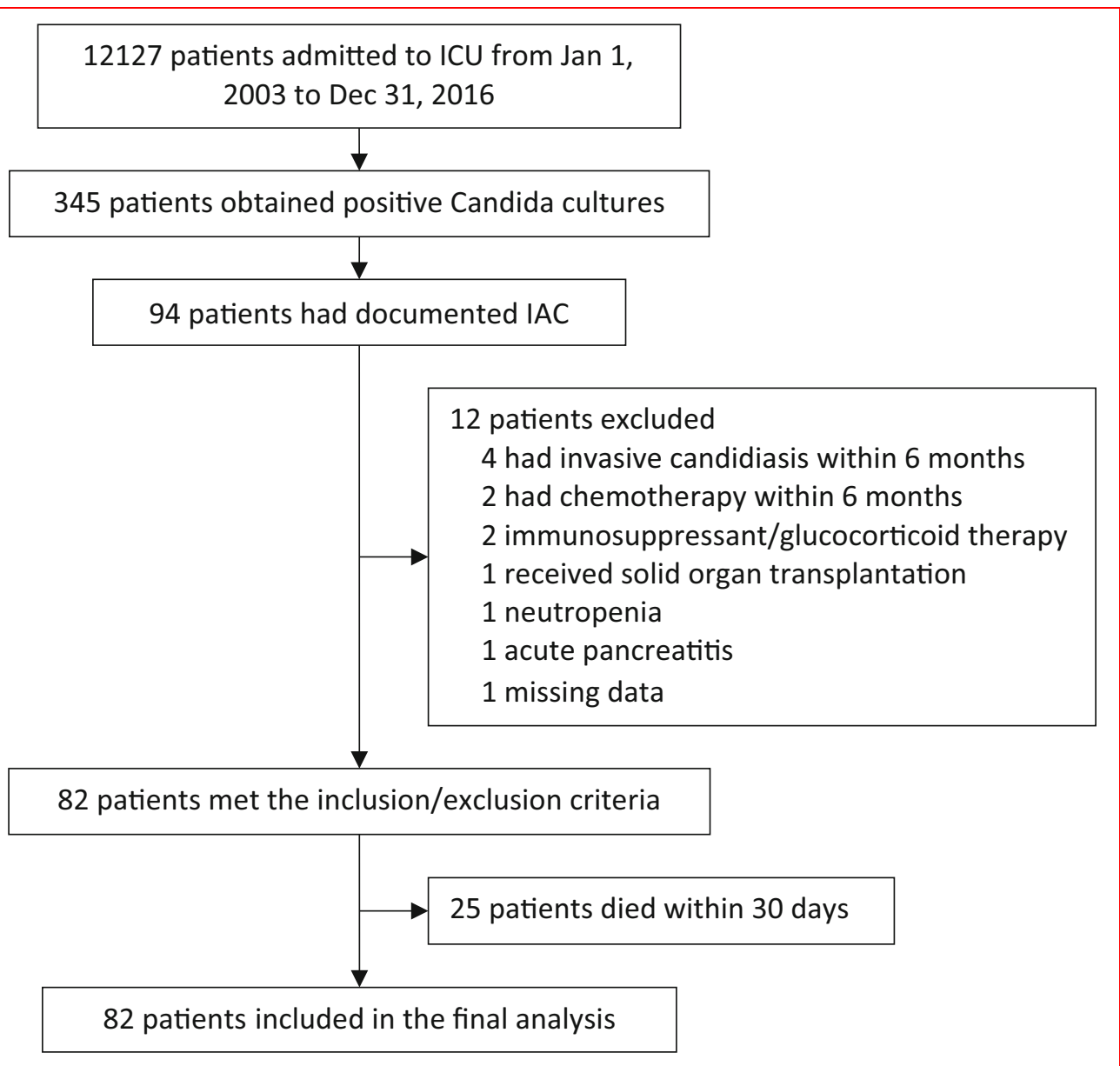

candidemia occurred in $70.7 \%$ and $20.7 \%$ of patients, respectively (Table 2 ).

\section{Treatment and outcomes}

Of the included patients, $53(64.6 \%)$ received appropriate source control and $44(53.7 \%)$ received appropriate antifungal therapy. The overall 30-day mortality was $30.5 \%$ $(25 / 82)$. One patient died of cerebrovascular event and the rest died of infection-related multiple organ failure. Comparison among groups showed that the 30-day mortality rate in patients with both appropriate source control and appropriate antifungal therapy was significantly lower than in those with neither appropriate treatment $(P<0.001)$ and those with only appropriate antifungal therapy $(P=0.003)$ (Table 3 and Fig. 2).

\section{Association between appropriate treatment and 30- day survival}

Univariable analyses identified 7 factors that might be associated with 30-day survival $(P<0.10)$, including age $>65$ years, SOFA score, septic shock, the year of IAC diagnosis, colonization by Candida species, appropriate source control, and appropriate antifungal therapy. Of these, SOFA score was excluded because of collinearity with septic shock; other 6 factors were included in the multivariable Cox proportional hazards model. The results showed that both appropriate source control (HR 0.09, 95\% CI $0.03-0.26 ; P<0.001)$ and appropriate antifungal therapy (HR $0.18,95 \%$ CI $0.07-0.47 ; P=0.001$ ) were the independent protective factors of 30-day survival in patients with IAC. We also analyzed the combined effect of appropriate treatment, and the results showed that the protective effects of appropriate source control and appropriate antifungal therapy were additive. When compared with patients with neither appropriate treatment, those with only appropriate antifungal therapy (HR 0.14, 95\% CI $0.04-0.55 ; P=0.005)$, only appropriate source control (HR 0.08, 95\% CI 0.02-0.30; $P<0.001$ ), and both (HR $0.02,95 \%$ CI $0.00-0.08 ; P<0.001$ ) had significantly improved 30-day survival (Table 4). 


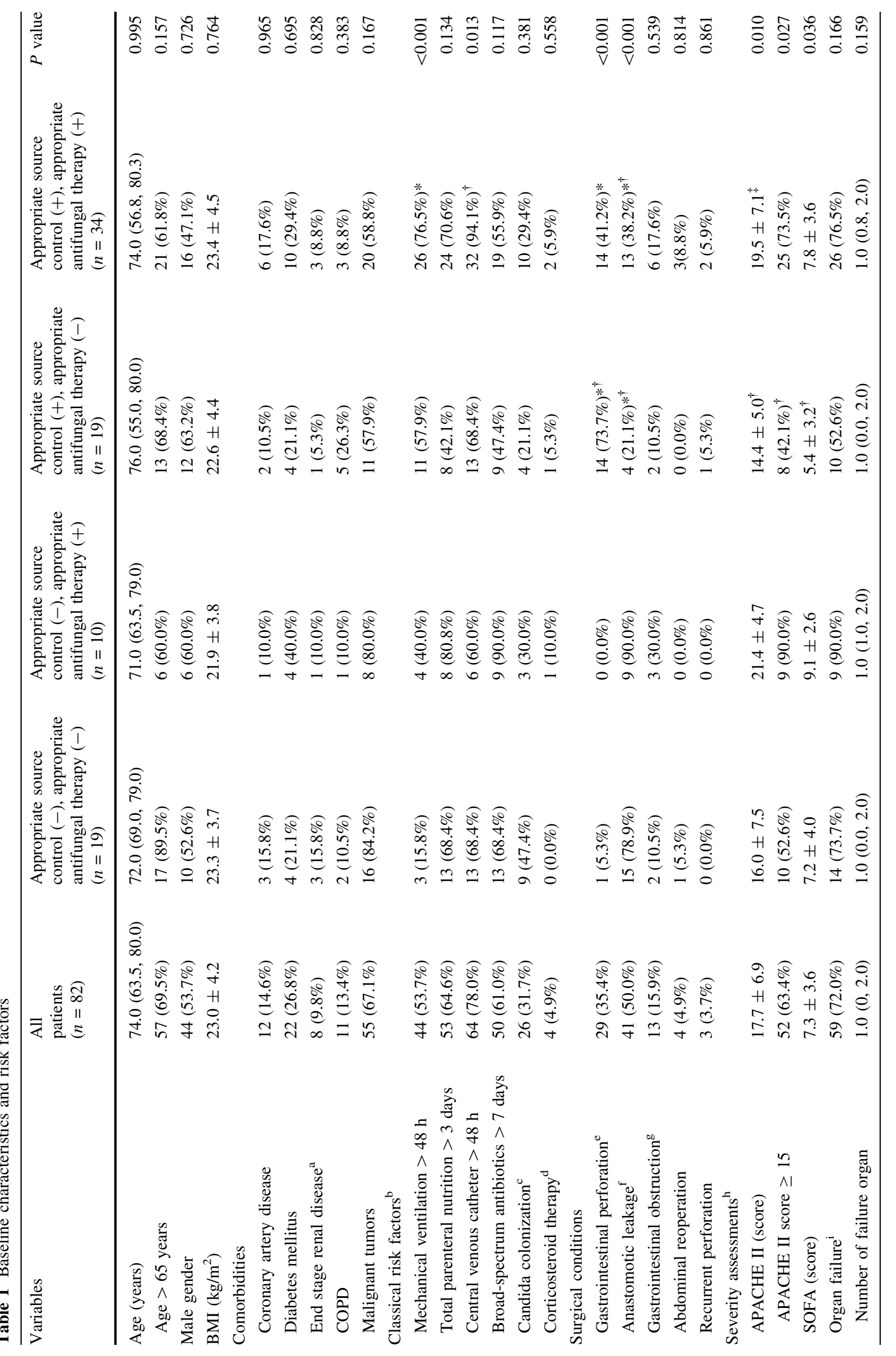




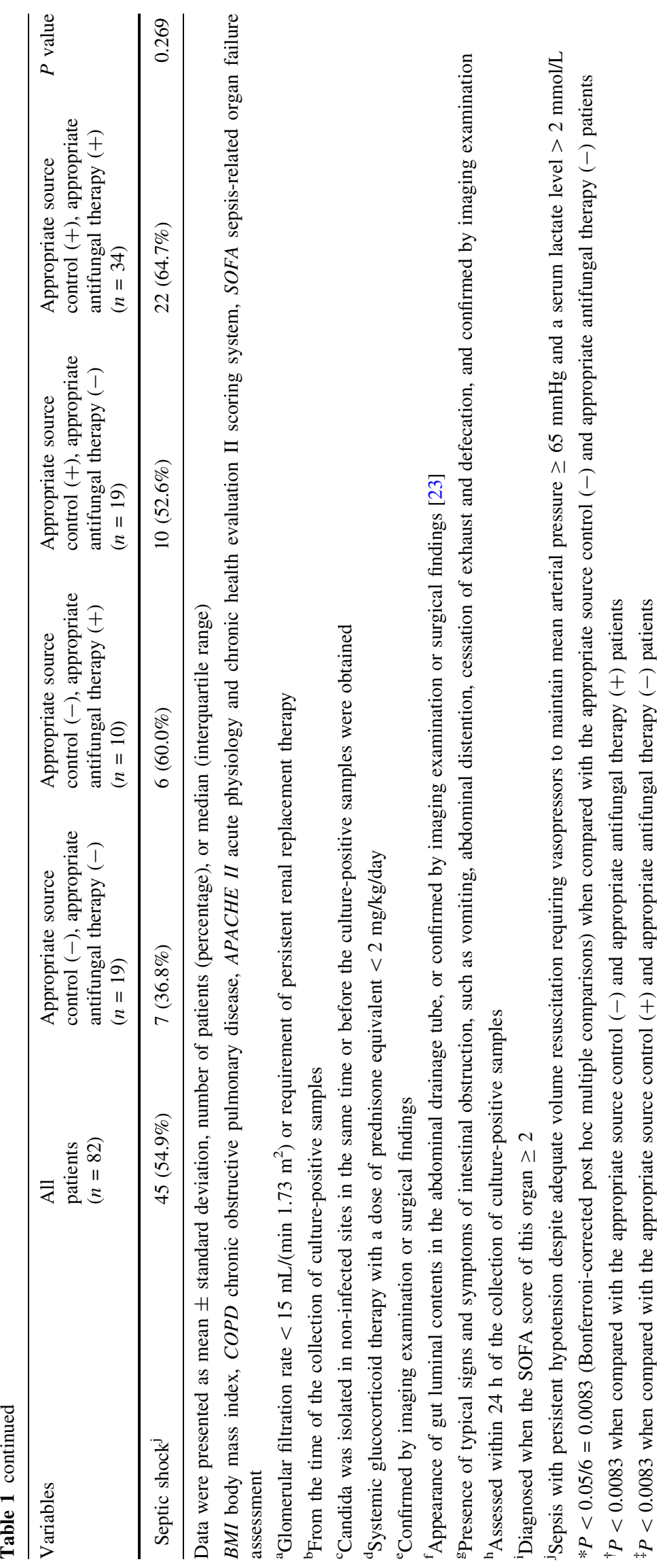




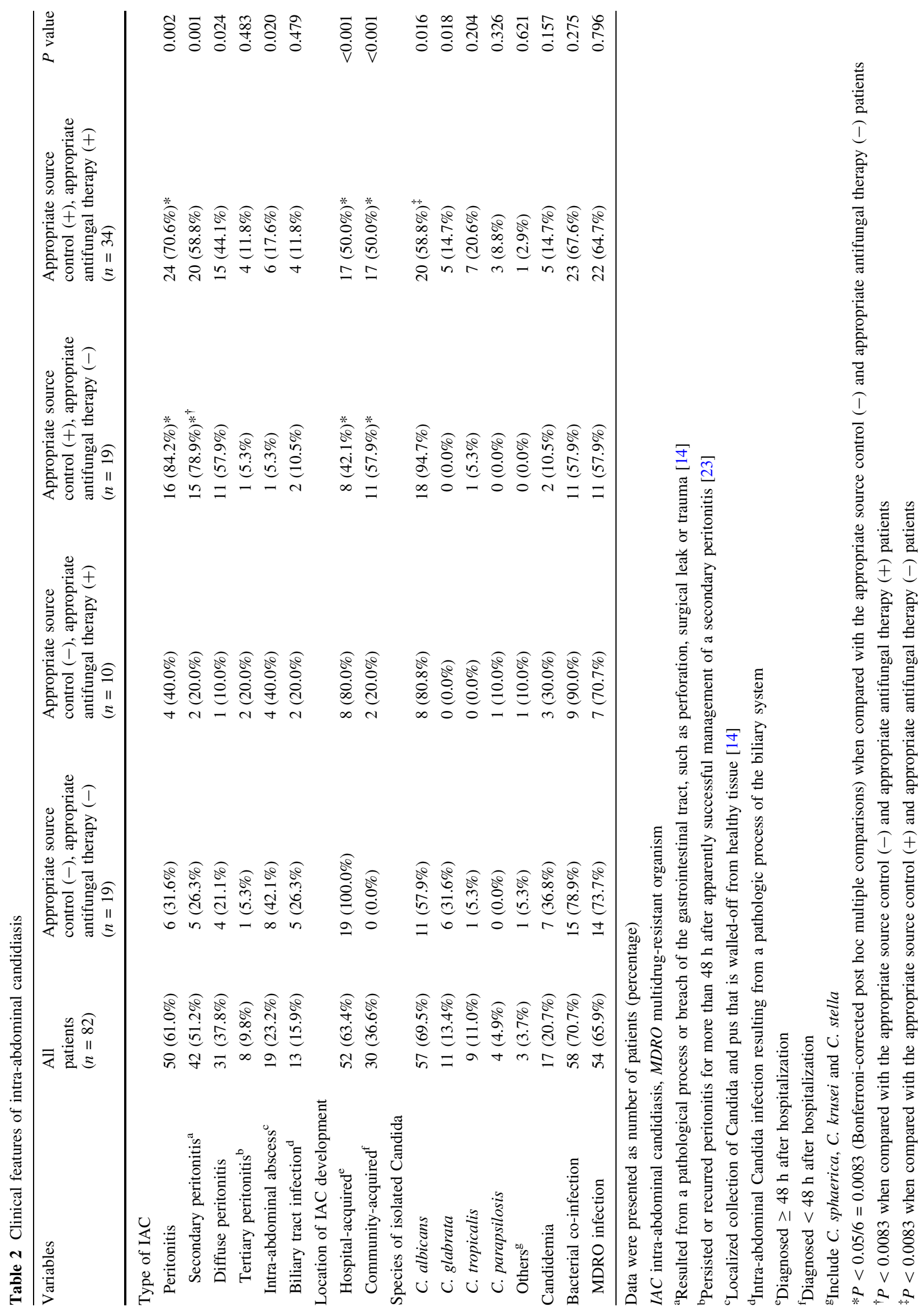




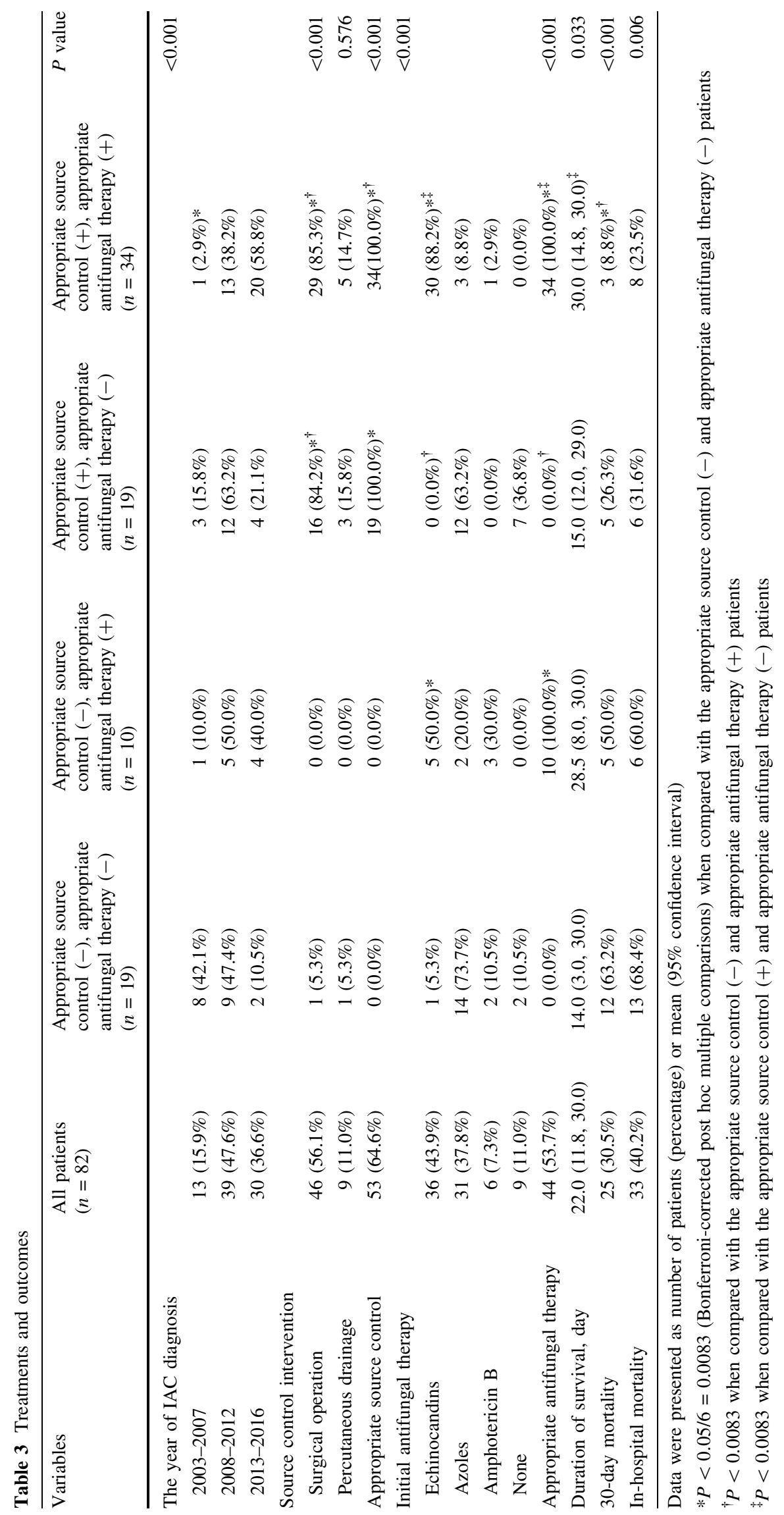




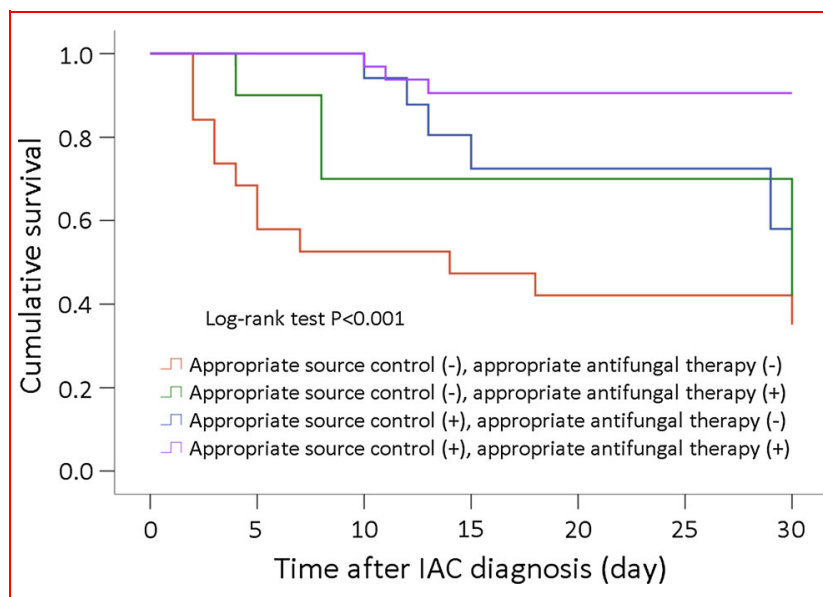

Fig. 2 The 30-day survival curve of patients with four combinations of appropriate treatments. Patients with both appropriate source control and appropriate antifungal therapy had significantly higher survival than those with neither appropriate treatment $(P<0.001)$ and those with only appropriate antifungal therapy $(P=0.003) . \quad P<0.05 / 6=0.0083$ were considered statistically significant (Bonferroni correction)

\section{Discussion}

Results of our study showed that the overall 30-day mortality was $30.5 \%$ in critically ill surgical patients with IAC; whereas appropriate source control and appropriate antifungal therapy were associated with improved 30-day survival, and the protective effects of the two appropriate treatments were additive. To our knowledge, this is the first study investigating the additive impact of appropriate treatments on the outcome of IAC patients.

In the present study, the target population was patients in a surgical ICU with documented IAC. These patients had high average APACHE II score, high proportion of septic shock and organ failure, and were at high risk of IACrelated death [9, 17]. Therefore, it is of high clinical significance to investigate the impact of appropriate treatments on the outcome in these patients.

There were three types of intra-abdominal infections leading to IAC in our patients, i.e., peritonitis $(61.0 \%)$, abdominal abscesses $(23.2 \%)$ and biliary tract infection (15.9\%). This study did not include patients with acute necrotizing pancreatitis, because it was difficult to obtain standard pathogenic specimens and there was no uniform criterion for adequate source control in those patients complicated with abdominal infections. As in other studies, C. albicans was the main pathogen of IAC in this study [1, 3]; Candida glabrata (13.4\%) and Candida tropicalis (11.0\%) were common in non-albicans Candida species.

For patients with IAC, the clinical signs and symptoms are usually not specific, and the definitive culture results are difficult to obtain. Therefore, the diagnosis of IAC and treatment initiation is often delayed, which might explain the reasons of poor outcome in these patient populations. In the present study, $30.5 \%$ of IAC patients died within 30 days, which was in line with the previously reported results $[6,24]$. The role of antifungal agents in the treatment of IAC remains somewhat controversial. For example, in a multicenter retrospective cohort study, Bassetti et al. [6] found that inadequate antifungal therapy (adequate therapy was define as susceptible and sufficient dose antifungal agents administered within $24 \mathrm{~h}$ from positive culture) was associated with 30-day mortality in IAC patients. In the study of Vergidis et al. [14], antifungal therapy was defined early when administered within 5 days of collecting culture-positive sample. Their results showed that early antifungal therapy was associated with better survival only for IAC stemming from gastrointestinal tract sources [14]. On the other hand, Lagunes et al. [15] reported that inadequate source control, but not inadequate antifungal therapy, was a risk factor for 30-day mortality in both ICU and non-ICU patients with IAC. It should be noted that, in the above studies, the effect of combined appropriate treatments (source control and antifungal therapy) had not been analyzed, and the effect of appropriate antifungal therapy need to be clarified further in surgical ICU patients.

In the present study, we defined interventions as early according to the same criteria of Vergidis et al. [14], i.e., those that were administered within 5 days of culturepositive sample collection. Our results showed that patients with higher APACHE II score, higher SOFA score and mechanical ventilation were more likely to receive appropriate antifungal therapy; similar phenomenon was also reported by others $[14,15,24]$. Despite of these, appropriate antifungal therapy remained as a protective factor of 30-day survival after correcting confounding factors in our patients. Furthermore, our results showed an additive effect of combined appropriate source control and appropriate antifungal therapy, i.e., those with both appropriate treatments had an even lower 30-day mortality. Therefore, combined appropriate treatments should begin as early as possible for surgical patients with IAC in the ICU.

Except the retrospective nature, there were some other limitations in our study. Firstly, the 2016 guideline recommends echinocandin susceptibility testing for patients who had prior echinocandin exposure or were infected with C. glabrata or C. parapsilosis [20], whereas such susceptibility testing was not routinely performed for isolated Candida species in our hospital. However, resistance to echinocandin-class drugs remains relatively low, i.e., less than 3\% of Candida albicans and most Candida species [25]. The multicenter study of Bassetti et al. [6] revealed that only $2 \%$ of Candida strains are resistant to echinocandin. Furthermore, our study excluded patients 
Table 4 Factors in association with 30-day survival (Cox proportional hazard model)

\begin{tabular}{|c|c|c|c|c|}
\hline & \multicolumn{2}{|l|}{ Univariate analysis } & \multicolumn{2}{|l|}{ Multivariate analysis $^{\mathrm{a}}$} \\
\hline & HR $(95 \% \mathrm{CI})$ & $P$ value & $\mathrm{HR}(95 \% \mathrm{CI})$ & $P$ value \\
\hline \multicolumn{5}{|l|}{ Separate effects of appropriate treatment } \\
\hline Appropriate source control & $0.21(0.90-0.48)$ & $<0.001$ & $0.09(0.03-0.26)$ & $<0.001$ \\
\hline Appropriate antifungal therapy & $0.30(0.13-0.71)$ & 0.006 & $0.18(0.07-0.47)$ & 0.001 \\
\hline Age $>65$ years & $3.20(0.96-10.68)$ & 0.059 & - & - \\
\hline Septic shock ${ }^{\mathrm{b}}$ & $2.03(0.88-4.71)$ & 0.099 & $10.97(3.54-33.93)$ & $<0.001$ \\
\hline Candida colonization $^{\mathrm{c}}$ & $2.11(0.96-4.62)$ & 0.063 & - & - \\
\hline \multicolumn{5}{|l|}{ The year of IAC diagnosis } \\
\hline 2013-2016 & 1.00 & & - & - \\
\hline 2008-2012 & $2.14(0.75-6.06)$ & 0.154 & - & - \\
\hline 2003-2007 & $4.33(1.41-13.24)$ & 0.010 & - & - \\
\hline \multicolumn{5}{|l|}{ Combined effect of appropriate treatment } \\
\hline \multicolumn{5}{|l|}{ Treatment combination } \\
\hline Appropriate source control $(-)$, appropriate antifungal therapy $(-)$ & 1.00 & & 1.00 & \\
\hline Appropriate source control $(-)$, appropriate antifungal therapy $(+)$ & $0.59(0.21-1.67)$ & 0.315 & $0.14(0.04-0.55)$ & 0.005 \\
\hline Appropriate source control $(+)$, appropriate antifungal therapy $(-)$ & $0.35(0.12-0.99)$ & 0.050 & $0.08(0.02-0.30)$ & $<0.001$ \\
\hline Appropriate source control $(+)$, appropriate antifungal therapy $(+)$ & $0.09(0.03-0.33)$ & $<0.001$ & $0.02(0.00-0.08)$ & $<0.001$ \\
\hline Age $>65$ years & $3.20(0.96-10.68)$ & 0.059 & - & - \\
\hline Septic shock ${ }^{\mathrm{b}}$ & $2.03(0.88-4.71)$ & 0.099 & $12.26(3.53-42.59)$ & $<0.001$ \\
\hline Candida colonization $^{\mathrm{c}}$ & $2.11(0.96-4.62)$ & 0.063 & - & - \\
\hline \multicolumn{5}{|l|}{ The year of IAC diagnosis } \\
\hline 2013-2016 & 1.00 & & - & - \\
\hline 2008-2012 & $2.14(0.75-6.06)$ & 0.154 & - & - \\
\hline 2003-2007 & $4.33(1.41-13.24)$ & 0.010 & - & - \\
\hline
\end{tabular}

$H R$ hazard ratio, $C I$ confidence interval, SOFA sepsis-related organ failure assessment

${ }^{a}$ Factors with a $P$ value of $<0.10$ in univariate analyses were included in multivariate model (backward). SOFA score was excluded because of collinearity with septic shock

${ }^{\mathrm{b}}$ Sepsis with persistent hypotension despite adequate volume resuscitation requiring vasopressors to maintain mean arterial pressure $\geq 65 \mathrm{mmHg}$ and a serum lactate level $>2 \mathrm{mmol} / \mathrm{L}$

${ }^{\mathrm{c}}$ Candida was isolated in non-infected sites in the same time or before the culture-positive samples were obtained

who received systemic antifungal therapy within 14 days. Therefore, the rate of echinocandin resistance might be very low in our patients. Secondly, because of the low incidence of IAC, we collected data over a 13-year period. The changes of routine practice during this long period might have confounded patients' outcomes. However, inclusion of the year of IAC diagnosis in the multivariate model did not change our results. Finally, because of the rarity of IAC cases, the sample size and the number of cases with positive events ( 25 deaths within 30 days) were relatively small in the present study, leaving a risk of estimation bias. However, with a backward elimination procedure, the factors remained significant in the multivariate model were no more than three; thus the "ten events per variable" rule was observed. This further confirmed the clinical significance of our results.

\section{Conclusion}

Our results showed that, in critically ill surgical patients with IAC, both appropriate source control and appropriate antifungal therapy were associated with reduced risk of mortality within 30 days, and the protective effects of two appropriate treatments were additive. Prospective trials are needed to verify these findings.

Acknowledgements The authors gratefully acknowledge Dr. Hai$\mathrm{Xia} \mathrm{Li} \mathrm{and} \mathrm{Dr.} \mathrm{Li-Ying} \mathrm{Sun} \mathrm{(Department} \mathrm{of} \mathrm{Clinical} \mathrm{Laboratory,}$ Peking University First Hospital, Beijing, China) for their microbiological consultations.

Author's contributions TY and SLL conceived of the study. TY, SLL and DXW participated in the study design and coordination of the study. TY and HLO contributed to data collection. TY and DXW performed data management. SNZ performed the statistical analysis. 
TY and LH drafted the manuscript. TY and DXW revised the manuscript. All authors had read and approved the final manuscript. DXW is the principal investigator and has overall responsibility for the study.

\section{Funding None.}

\section{Compliance with ethical standards}

Conflict of interest The authors declare that they have no conflict of interests.

Consent to participate Because of the retrospective and observational nature of the study, the local Ethics Committee agreed to exempt written informed consent.

Ethics approval The original study protocol was approved by the Clinical Research Ethics Committee of Peking University First Hospital (2017-1303).

Open Access This article is licensed under a Creative Commons Attribution 4.0 International License, which permits use, sharing, adaptation, distribution and reproduction in any medium or format, as long as you give appropriate credit to the original author(s) and the source, provide a link to the Creative Commons licence, and indicate if changes were made. The images or other third party material in this article are included in the article's Creative Commons licence, unless indicated otherwise in a credit line to the material. If material is not included in the article's Creative Commons licence and your intended use is not permitted by statutory regulation or exceeds the permitted use, you will need to obtain permission directly from the copyright holder. To view a copy of this licence, visit http://creativecommons. org/licenses/by/4.0/.

\section{References}

1. Aguilar G, Delgado C, Corrales I et al (2015) Epidemiology of invasive candidiasis in a surgical intensive care unit: an observational study. BMC Res Notes 8:491

2. Leroy O, Gangneux JP, Montravers P et al (2009) Epidemiology, management, and risk factors for death of invasive Candida infections in critical care: a multicenter, prospective, observational study in France (2005-2006). Crit Care Med 37:1612-1618

3. de Ruiter J, Weel J, Manusama E et al (2009) The epidemiology of intra-abdominal flora in critically ill patients with secondary and tertiary abdominal sepsis. Infection 37:522-527

4. Montravers P, Dupont H, Gauzit R et al (2006) Candida as a risk factor for mortality in peritonitis. Crit Care Med 34:646-652

5. Dupont H, Paugam-Burtz C, Muller-Serieys C et al (2002) Predictive factors of mortality due to polymicrobial peritonitis with Candida isolation in peritoneal fluid in critically ill patients. Arch Surg 137:1341-1346

6. Bassetti M, Righi E, Ansaldi F et al (2015) A multicenter multinational study of abdominal candidiasis: epidemiology, outcomes and predictors of mortality. Intensive Care Med 41:1601-1610

7. Rebolledo M, Sarria JC (2013) Intra-abdominal fungal infections. Curr Opin Infect Dis 26:441-446

8. Montravers P, Leroy O, Eckmann C (2015) Intra-abdominal candidiasis: it's still a long way to get unquestionable data. Intensive Care Med 41:1682-1684
9. Montravers P, Dupont H, Eggimann P (2013) Intra-abdominal candidiasis: the guidelines-forgotten non-candidemic invasive candidiasis. Intensive Care Med 39:2226-2230

10. Kollef M, Micek S, Hampton N et al (2012) Septic shock attributed to Candida infection: importance of empiric therapy and source control. Clin Infect Dis 54:1739-1746

11. Morrell M, Fraser VJ, Kollef MH (2005) Delaying the empiric treatment of candida bloodstream infection until positive blood culture results are obtained: a potential risk factor for hospital mortality. Antimicrob Agents Chemother 49:3640-3645

12. Grim SA, Berger K, Teng C et al (2012) Timing of susceptibilitybased antifungal drug administration in patients with Candida bloodstream infection: correlation with outcomes. J Antimicrob Chemother 67:707-714

13. Ostrosky-Zeichner L, Kullberg BJ, Bow EJ et al (2011) Early treatment of candidemia in adults: a review. Med Mycol 49:113-120

14. Vergidis P, Clancy CJ, Shields RK et al (2016) Intra-abdominal candidiasis: the importance of early source control and antifungal treatment. PLoS ONE 11:e0153247

15. Lagunes L, Rey-Perez A, Martin-Gomez MT et al (2017) Association between source control and mortality in 258 patients with intra-abdominal candidiasis: a retrospective multi-centric analysis comparing intensive care versus surgical wards in Spain. Eur J Clin Microbiol Infect Dis 36:95-104

16. Dimopoulos G, Matthaiou DK, Righi E et al (2017) Elderly versus non-elderly patients with intra-abdominal candidiasis in the ICU. Minerva Anestesiol 83:1126-1136

17. Bassetti M, Marchetti M, Chakrabarti A et al (2013) A research agenda on the management of intra-abdominal candidiasis: results from a consensus of multinational experts. Intensive Care Med 39:2092-2106

18. National Committee for Clinical Laboratory Standards (NCCLS) (1997) Reference method for broth dilution antifungal susceptibility testing of yeasts; Approved standard-second edition. NCCLS document M27-A NCCLS, Wayne

19. Solomkin JS, Ristagno RL, Das AF et al (2013) Source control review in clinical trials of anti-infective agents in complicated intra-abdominal infections. Clin Infect Dis 56:1765-1773

20. Pappas PG, Kauffman CA, Andes DR et al (2016) Clinical practice guideline for the management of candidiasis: 2016 update by the Infectious Diseases Society of America. Clin Infect Dis 62:e1-50

21. Bingold TM, Lefering R, Zacharowski $\mathrm{K}$ et al (2015) Individual organ failure and concomitant risk of mortality differs according to the type of admission to ICU-a retrospective study of SOFA score of 23,795 patients. PLoS ONE 10:e0134329

22. Singer M, Deutschman CS, Seymour CW et al (2016) The third international consensus definitions for sepsis and septic shock (Sepsis-3). JAMA 315:801-810

23. Mazuski JE, Tessier JM, May AK et al (2017) The surgical infection society revised guidelines on the management of intraabdominal infection. Surg Infect (Larchmt) 18:1-76

24. Maseda E, Rodríguez-Manzaneque M, Dominguez D et al (2016) Intraabdominal candidiasis in surgical ICU patients treated with anidulafungin: a multicenter retrospective study. Rev Esp Quimioter 29:32-39

25. Perlin DS (2015) Echinocandin resistance in Candida. Clin Infect Dis 61(Suppl 6):S612-S617

Publisher's Note Springer Nature remains neutral with regard to jurisdictional claims in published maps and institutional affiliations. 